\title{
A SIMILARITY SOLUTION TO A NONLINEAR DIFFUSION EQUATION OF THE SINGULAR TYPE: A UNIFORMLY VALID SOLUTION BY PERTURBATIONS*
}

BY

\author{
D. K. BABU and M. Th. VAN GENUCHTEN
}

Princeton University

\begin{abstract}
The one-dimensional nonlinear diffusion equation is solved by a perturbation technique. It is assumed that the diffusivity varies as a nonnegative power of the concentration, while the concentration at the supply surface varies as another power of time. The resulting similarity solution that has been derived via a perturbation scheme remains valid for all times and all distances. Explicit series formulae are also derived for the location of the concentration front. Since diffusivity vanishes at zero concentration, the study here pertains to a singular problem.
\end{abstract}

Introduction. The nonlinear diffusion equation, and certain other types of parabolic equations associated with it, are appearing with increasing frequency in many problems of the physical and environmental sciences. A special case of the following problem, admitting of a similarity solution, will be solved in the next sections:

$$
\begin{gathered}
\frac{\partial}{\partial x}\left[D(\theta) \frac{\partial \theta}{\partial x}\right]=\frac{\partial \theta}{\partial t}, \quad 0 \leq \theta \leq 1, t>0,0<x<F(t), F(t) \text { unknown, } \\
D(0)=0, D(1)=1, F(0)=0 ; \quad x=0, \theta=f(t) ; \quad t=0, \theta=0 ; \\
x \geq F(t), \theta \equiv 0 \quad \text { and } \quad D(\theta) \frac{\partial \theta}{\partial x} \equiv 0 .
\end{gathered}
$$

Here $x=F(t)$ is the distance of the advancing "concentration or diffusing front". Throughout this work, it is assumed that all variables and functions have been rendered dimensionless by appropriate normalizations. $x$ and $t$ are respectively the space and time variables. $\theta$ may be identified with the concentration of a solution, moisture content of a partially saturated soil, temperature of a conducting medium, etc.

The relation $D(0)=0$ is an important one here, and mention must be made of its role in structuring a solution to this problem. It will be noticed that $D(\theta)$ is the (nonlinear) coefficient of the highest derivative $\left(\partial^{2} \theta / \partial x^{2}\right)$ in the differential equation under study. It is well known that the points at which such coefficients vanish are generally associated with "singular" points of the solution function $\theta(x, t)$. Shampine [1] investigated in detail a problem of this type. In his paper, the special boundary conditions $x=0, \theta=1, x=\infty \theta=$ 0 led to an ordinary differential equation in the similarity variable $s=(x / \sqrt{ } t)$. He used the term "singular problem" to denote the cases that arise out of the special condition $D(0)=$ 0 .

* Received December 27, 1977; revised version received August 4, 1978. Work on this paper was supported by the National Science Foundation under grants number ENG 7702013, ENG 7724979. 
The mathematical pecularities, as well as the physical characteristics, of the resulting solution profiles $\theta(x, t)$ are worth mentioning at this stage. These are: (a) excluding any unbounded solutions, there still exists the possibility of singularities in the derivatives of $\theta$; (b) although the problem could be formulated, as usual, in the semi-infinite domain $0<x$ $<\infty, \theta \rightarrow 0$ as $x \rightarrow \infty$, the (strong) possibility exists that the profile $\theta(x, t)$ actually terminates at a finite distance, called the diffusing front; (c) to begin with, the location of this front at $F(t)$ is unknown; (d) it is highly likely that the gradient at the front is unbounded: $x=F(t), \partial \theta / \partial x=-\infty$, so that the "sharp front" occurrence is observed; and (e) in many applications, the function $D(\theta)$ is an extremely rapidly varying function of $\theta$. It is needless to state here that accurate profile evaluation and numerical computations would be rendered more difficult by each of the above pecularities (a)-(e).

A basic purpose of the present investigation is to evolve a constructive technique that is capable of generating an accurate solution profile, in spite of the above complications. Indeed, it will be seen in later sections of this paper that the complexity $(e)$ is turned around to advantage, and the construction of the solution specifically depends on the use of rapid variation in $D(\theta)$.

Since $D(\theta)$ is allowed to vanish at $\theta=0$ in the above equation, it is expected that the profile " $\theta$ versus $x$ " is finite in extent, terminating abruptly at the diffusing front (Shampine [1]). In many applications, $D(\theta)$ is known to be a rapidly varying function of $\theta$. In soil moisture problems, $D(\theta)$ is frequently assumed to be an exponential function of $\theta$ (Reichardt et al. [2]). Often, it is assumed that $f(t) \equiv 1$; the solution then will depend on a similarity variable $\eta=(x / \sqrt{ } t)$. Assuming $D(\theta)=\theta^{n}$, Pattle [3] constructed an instantaneous point-source type of solution for the nonlinear equation (see note at the end). Aronson [4] investigated certain regularity properties of solutions with finite profile termination. Solutions exhibiting "square root of time" dependence, and their asymptotic behavior, have been studied by Peleiter [5], Atkinson and Jones [6], and Pimbeley [7]. Most of these studies deal with the existence and uniqueness aspects of the solutions.

Horizontal absorption of water by dry and partially dry soils is extensively modelled on the diffusion equation. Survey work by Philip $[8,9]$ gives an idea of the importance attached to equations of this type in soil water transport problems. The high nonlinearities inherent in these equations have naturally generated a vast body of computer solutions based upon numerical schemes. Numerical solutions of the above equations, subject to general initial and boundary conditions, have been obtained by several authors. In general, finite difference and finite element schemes have gained wide acceptance in practice. Numerous references may be found in the articles by Ashcroft et al. [10], Neumann [11], Hayhoe [12] and van Genuchten [13]. By contrast, analytic and quasianalytic solutions of a constructive nature are few in number. Brutsaert [14] presented a solution with finitely terminating profiles, referring to an earlier work of Heaslet and Alksne [15]. Parlange [16] obtained elegant results by a method of iterations resembling the Picard iterates. Babu $[17,18,19]$ applied perturbation techniques to achieve a constructive solution that is capable of yielding quantitative information in a straightforward and reliable fashion, for the special case of $f(t) \equiv 1$. In a subsequent joint work, Parlange and Babu $[20,21]$ exhibited the essential identity of the solutions constructed via perturbation, iteration and optimization techniques.

In what follows, it will be assumed that $f(t)=t^{m}$ and $D(\theta)=\theta^{n}(n \geq 0)$. The solution mechanism presented below is valid for values of $m$ and $t$ in the ranges $-1 /(n+1)<m<$ $+\infty, m \neq 0 ; 0 \leq t<\infty$. The case $m=0$ requires only minor adjustments in the computational detail. However, the case of $0<m<+\infty, 0 \leq t \leq 1$ alone is worked out in 
detail here (see note at the end). The case $n=1$ embraces a very large body of studies in shallow groundwater flows described by the well-known Dupuit Forcheimer theory [22].

The equations. It is therefore required to solve

$$
\frac{\partial}{\partial x}\left[\theta^{n} \frac{\partial \theta}{\partial x}\right]=\frac{\partial \theta}{\partial t}, \quad 0<t<1, \quad 0<x<F(t), \quad n \geq 0
$$

subject to the conditions

$$
\begin{aligned}
\theta & =t^{m}, x=0, m>0, \\
\theta & =0, t=0, \\
\theta & =0, x \geq F(t), \\
\theta^{n} & \cdot \frac{\partial \theta}{\partial x}=0, x \geq F(t) .
\end{aligned}
$$

Condition $(2 c)$ states that the $\theta(x, t)$ solution profile "hits" the $x$-axis at (an unknown) distance $F(t)$, forming a "diffusing front" there. And, since the flux also vanishes according to $(2 d)$, it follows that no disturbances are propagated into the medium beyond this front. Part of the solution process will thus be devoted to the determination of the unknown function $F(t)$.

Since $\theta=0$ at $x=F(t)$, condition ( $2 d)$ may in fact be an identity, unless $|\partial \theta / \partial x|=\infty$. Hence, from a computational point of view, this condition may not often yield much useful information. However, an integration of Eq. (1), utilizing $(2 a-(2 d)$, leads to the relation:

$$
\begin{aligned}
\int_{0}^{F(t)} x \cdot \frac{\partial \theta}{\partial t} \cdot d x & =\int_{0}^{F(t)} x \cdot \frac{\partial}{\partial x}\left(\theta^{n} \frac{\partial \theta}{\partial x}\right) \cdot d x \\
& =\left.x \cdot \theta^{n} \frac{\partial \theta}{\partial x}\right|_{0} ^{F(t)}-\int_{0}^{F(t)} 1 \cdot \theta^{n} \frac{\partial \theta}{\partial x} \cdot d x=\int_{\theta=0}^{\theta=t^{m}} \theta^{n} d \theta \\
& =\left.\frac{\theta^{n+1}}{(n+1)}\right|_{0} ^{t^{m}} \frac{d}{d t} \int_{0}^{F(t)}, \text { so that } x \cdot \theta(x, t) \cdot d x=\epsilon t^{m / \epsilon}
\end{aligned}
$$

where $\epsilon \equiv 1 / n+1$. One final integration yields

$$
\int_{0}^{F(t)} x \cdot \theta(x, t) \cdot d x=\frac{\epsilon^{2} \cdot t^{1+m / \epsilon}}{m+\epsilon} .
$$

Next, elementary estimates, based upon (3), lead to an explicit determination of the parameter of perturbation for this problem. This parameter is $\epsilon \equiv 1 /(n+1)$. Neglecting the time dependency for the moment, it follows from (3) that $x^{2}=O\left(\epsilon^{2}\right)$, showing that the entire phenomenon is of a boundary-layer nature, if $\epsilon$ is small in some sense. Some general comments on the implications of (3) are to be found in the Appendix.

The following transformations reduce (1) to an ordinary differential equation:

$$
\begin{gathered}
\epsilon=1 /(n+1) ; \eta=(x / \epsilon) \cdot(m / \phi(\epsilon, m))^{1 / 2} \cdot\left(1 / t^{m n+1}\right)^{1 / 2} ; \\
\theta=t^{m} \cdot[V(\eta ; \epsilon ; m)]^{\epsilon} ; \\
\left(\frac{d^{2} V}{d \eta^{2}}\right)=\left[\epsilon V^{\epsilon}-\left(\frac{\eta}{2}\right)[1-\epsilon(1-1 / m)] \cdot\left(\frac{d V^{\epsilon}}{d \eta}\right)\right] \cdot \phi(\epsilon, m)
\end{gathered}
$$


along with the conditions

$$
\text { at the origin } \eta=0, V=1 \text {, }
$$

$$
\text { at the front } \eta=1 \text {, concentration } V=0 \text { and flux } d V / d \eta=0 \text {. }
$$

Here the unknown parametric function $\phi(\epsilon, m)$ defines the position of the unknown front in the physical coordinates, via

$$
\eta=1, x=F(t)=\epsilon \cdot\left(t^{m n+1}\right)^{1 / 2} \cdot(\phi(\epsilon, m) / m)^{1 / 2} .
$$

The zero flux condition implicit in (3) becomes

$$
\left(\int_{0}^{1} V^{\epsilon} \cdot \eta \cdot d \eta\right) \cdot \phi(\epsilon ; m) \cdot(1+\epsilon / m)=1 .
$$

A solution to the equations (5)-(9) will be derived in the next sections. Through back substitutions in (4), the corresponding solution to (1) and (2) may be achieved in the final form. In passing, it may be mentioned that whenever $V(\eta)$ is bounded, the initial condition $(2 b)$ is automatically satisfied (Definition (4)).

The parameter $\epsilon$. The identification and the definition of the parameter $\epsilon$ is pivotal to the scheme of solution proposed in this paper. It will be noticed that the original problem, as postulated in (1)-(2), did not explicitly show a parameter for perturbation. But from (3), it is apparent that the profile lengths are of the order of magnitude of $\epsilon$. In a sense, therefore, the number $\epsilon$ "calibrates" the profile sizes. It is this fundamental property of $\epsilon$ that suggests the perturbation parameter should indeed be identified with $\epsilon$. The intimate connection of $\epsilon$ to the "physics" of the transport parameter $D(\theta)$ makes one expect that a solution scheme that utilizes $\epsilon$ should prove quite efficient. It turns out that this is indeed the case.

Existing methods of solution. The differential equation (5) may be written in the general form

$$
d^{2} v / d \eta^{2}=g\left(\eta ; v ; v^{\epsilon} ; d v / d \eta ; \epsilon\right), 0<\eta<1,
$$

where the nonlinear function $g$ becomes unbounded as $\eta \rightarrow 1$ (or $v \rightarrow 0$ ). In other words, $g$ is not a regular function of its arguments. The presence of such singularities will generally render any perturbation scheme into a singular type. The presence of unbounded derivatives $(d v / d \eta)$, and the terms in $v^{\epsilon}$ for small values of $v$, definitely lead to a breakdown of regular, straightforward expansions.

Now, if $g$ were an analytic function of its arguments, the usual (existing) procedures indicate a power series expansion in $\epsilon$ for both $v$ and $g$. Often, there will arise also the need to match the inner and outer solutions asymptotically. In any case, the solution structure to be tried will be as follows:

$$
v=\sum_{0}^{\infty} \epsilon^{n} \cdot v_{n}(\eta, \zeta)
$$

where $\eta$ is the outer variable and $\zeta$ a scaled variable defined in terms of $\eta$. The solution mechanism is established by equating coefficients of like powers of $\epsilon$, after substituting (B) into (A) and the associated boundary/initial conditions. A judicious choice for the structure of $\zeta$ is further expected to eliminate a breakdown of the resultant solution structure at the possible singularities. At each step in the solution computation, the 
structures of $\zeta$ and $v_{n}$ are adjusted so that singular or "secular" terms are eliminated. Methods of matched asymptotic expansions, strained coordinates, strained parameters, averaging techniques, composite expansions and the methods associated with the names of Krylov and Bogoliuboff, Lighthill, and others, are of this form. It is also customary to employ phase-plane and limit cycle analysis in many practically significant (autonomous) systems of equations. References may be found in the works of O'Malley [24], Krylov and Bogoliuboff [25], Nayfeh [23], van Dyke [26], and AMS Proceedings [27].

It is, of course, possible that such methods may not always succeed, even though the right-hand sides of (A) are analytic functions of their arguments. In the present case, it is apparent that a structure of the type (B) would not be suitable for solving (A). The reasons are clear: $g$ is singular at $\eta=1$, and $v^{\epsilon}$-expansions in power of $\epsilon$ break down as $v \rightarrow 0$. In order to deal with these complications and with a view to specially accommodating terms of the form $v^{\epsilon}$ in the governing differential equation, it was decided to try a solution in the form

$$
\left.v=\left[G_{0}(\eta)\right]^{\lambda(\epsilon ; \eta}\right) \cdot H(\epsilon ; \eta)
$$

where the functions $\lambda(\epsilon ; \eta)$ and $H(\epsilon ; \eta)$ have expansions in powers of $\epsilon$, and where $G_{0}(\eta)$ represents the starter solution in some sense. In this respect, the procedure outlined here is somewhat different from other standard methods in existence. The specific structure for $v(\eta ; \epsilon)$ as postulated in (C), is necessary to eliminate the singular or secular terms that result when expanding the right-hand side of (A) in a straightforward fashion.

The perturbation scheme. To begin with, since $\epsilon=1 /(n+1)$ is generally small in applications, a perturbation solution to (5)-(9) is attempted in the regular and straightforward form:

$$
\begin{gathered}
V(\eta ; \epsilon ; m)=V_{0}+\epsilon V_{1}+\epsilon^{2} V_{2}+\cdots, \\
\phi(\epsilon, m)=\phi_{0}+\epsilon \phi_{1}+\epsilon^{2} \phi_{2}+\cdots,
\end{gathered}
$$

where the $V_{n}$ 's are functions of $\eta$ and $m$, and the $\phi_{n}$ 's depend on $m$. The standard procedure is to substitute $\left(\mathrm{A}^{\prime}\right)$ and $\left(\mathrm{B}^{\prime}\right)$ into (5)-(9), collect the powers of $\epsilon$, and successively equate to zero the coefficients of these powers of $\epsilon$ (Nayfeh [23]). Although this procedure yields the starter terms

$$
V_{0}=1-\eta, \phi_{0}=2,
$$

it is immediately noticed that the next approximations $V_{1}, V_{2}, \cdots$ contain singular, or secular, components like $\ln (1-\eta), 1 /(n-1), \cdots$ The singularity keeps growing and the validity of the solution in (A) becomes seriously impaired, especially near $\eta=1$ (front). A fundamental source of this singularity in the solution process is the need for expansions for quantities like $V^{\epsilon},\left(d V^{\epsilon} / d \eta\right)$, in powers of $\epsilon\left(V^{\epsilon} \equiv 1+\epsilon \ln V+(\epsilon \ln V)^{2} / 2 !+\cdots\right)$. Such a process naturally leads to logarithmic and derivative singularities. In order to neutralize these singularities, and with a view to obtaining a uniformly valid solution, it appears that a correct structure for the solution would have to be tried in the form

$$
V=(1-\eta)^{1+\epsilon \lambda_{1}+\epsilon^{2} \lambda_{2}+\cdots}\left(1+\epsilon V_{1}+\epsilon^{2} V_{2}+\cdots\right)
$$

and

$$
\phi=\phi_{0}+\epsilon \phi_{1}+\epsilon^{2} \phi_{2}+\cdots
$$


Here, $\lambda_{1}, \lambda_{2}, \cdots$ are in general assumed to be functions of $\eta$, and will be specifically structured so as to remove the resultant singularities in the differential equations for the $V_{n}$ 's. In particular, it will turn out that the $\lambda_{n}$ 's are merely numerical constants.

Eqs. (10), (11), and (5)-(9) lead to the following sequence of problems:

$\epsilon^{0}$ terms;

$$
\begin{gathered}
\phi_{0}=2, \\
\left(d^{2} / d \eta^{2}\right)(1-\eta)=0 ;
\end{gathered}
$$

$\epsilon^{1}$ terms:

$$
\begin{gathered}
\left(\frac{d^{2}}{d \eta^{2}}\right)\left[(1-\eta)\left(V_{1}+\lambda_{1} \cdot \ln (1-\eta)\right)\right]=\phi_{0}\left(1+\frac{1}{2(1-\eta)}\right), \\
\eta=0, V_{1}=0, \\
\eta=1,\left|V_{1}\right|<\infty ;
\end{gathered}
$$

$\epsilon^{2}$ terms:

$$
\phi_{1}=3-2 / m,
$$

$$
\begin{gathered}
\left(\frac{d^{2}}{d \eta^{2}}\right)\left[\left(1-\eta\left(\left[V_{2}+\lambda_{2} \cdot \ln (1-\eta)+\lambda_{1} \cdot V_{1} \cdot \ln (1-\eta)+\lambda_{1}^{2} \cdot \ln ^{2}(1-\eta) / 2\right]\right]\right.\right. \\
=\phi_{0}\left[\ln (1-\eta)-\left(\frac{n}{2}\right)\left[(1-1 / m) /(1-\eta)+\left(\frac{d}{d \eta}\right)\left(V_{1}+\lambda_{1} \ln (1-\eta)\right.\right.\right. \\
\left.\left.\left.+\frac{1}{2} \cdot \ln ^{2}(1-\eta)\right)\right]\right]+\phi_{1}\left(1+\frac{\eta}{2(1-\eta)}\right), \\
\eta=0, V_{2}=0, \\
\eta=1,\left|V_{2}\right|<\infty ;
\end{gathered}
$$

$\epsilon^{3}$ terms:

$$
\phi_{2}=14 / 3-3 / m+2 / m^{2},
$$

$\left(\frac{d^{2}}{d \eta^{2}}\right)\left[(1-\eta)\left[V_{3}+\lambda_{3} \cdot \ln (1-\eta)\right]+(1-\eta) \cdot P_{1}\right]=\phi_{0} \cdot P_{2}+\phi_{1} \cdot P_{3}+\phi_{2} \cdot P_{4}$,

$$
\begin{gathered}
\eta=0, V_{3}=0, \\
\eta=1,\left|V_{3}\right|<\infty,
\end{gathered}
$$$$
P_{1}=\ln (1-\eta) \cdot\left[\lambda_{1} V_{2}+\lambda_{2} V_{1}+\left(\lambda_{1}^{2} V_{1} / 2\right) \cdot \ln (1-\eta)+\lambda_{1} \lambda_{2} \ln (1-\eta)+\left(\lambda_{1}^{3} / 6\right)\right.
$$$$
\left.\cdot \ln ^{2}(1-\eta)\right]
$$$$
P_{2}=V_{1}+\lambda_{1} \cdot \ln (1-\eta)+1 / 2 \cdot \ln ^{2}(1-\eta)
$$$$
-(\eta / 2) \cdot\left(\frac{d}{d \eta}\right)\left[V_{2}-V_{1}^{2} / 2+V_{1} \cdot \ln (1-\eta)+\lambda_{2} \cdot \ln (1-\eta)\right.
$$$$
+\lambda_{1} \cdot \ln ^{2}(1-\eta)+\frac{1}{8} \cdot \ln ^{3}(1-\eta)-(1-1 / m) \cdot\left(V_{1}+\lambda_{1} \cdot \ln (1-\eta)+\frac{1}{2} \cdot \ln ^{2}(1-\eta)\right],
$$ 


$$
\begin{gathered}
P_{3}=\ln (1-\eta)-(\eta / 2) \cdot\left[(1-1 / m) /(1-\eta)+\left(\frac{d}{d \eta}\right)\left[V_{1}+\lambda_{1} \ln (1-\eta)+1 / 2 \cdot \ln ^{2}(1-\eta)\right]\right], \\
P_{4}=1+\eta / 2(1-\eta) .
\end{gathered}
$$

Next, in (13), (14), (15), $\cdots$, singularities and secular terms will be eliminated if

$$
\lambda_{1}=1, \lambda_{2}=1, \lambda_{3}=1, \cdots,
$$

The approximations are given as

$$
\begin{gathered}
V_{1}=-\eta / 2 ; V_{2}=(1 / m-1 / 12) \eta-\eta^{2} / 12, \\
V_{3}=\left(2 /(3 m)-1 / m^{2}-3 / 16\right) \eta+(1 /(6 m)+1 / 16) \eta^{2}-5 \eta^{3} / 144, \\
\quad \phi_{0}=2 ; \phi_{1}=3-2 / m ; \phi_{2}=14 / 3-3 / m+2 / m^{2}, \ldots .
\end{gathered}
$$

It follows from (10), and the above relations, that $d V / d \eta=0$ at $\eta=1$. Thus, the condition of zero flux at the front is also satisfied (Eqs. (2d), (4) and (10)).

The solution. To the third-order terms, then, the solution would be given by

with

$$
\begin{aligned}
& \phi(x, t)=t^{m} \cdot V^{\epsilon}=t^{m} \cdot(1-\eta)^{\epsilon+\epsilon^{2}+\epsilon^{3}} \cdot\left(1+\epsilon V_{1}+\epsilon^{2} V_{2}+\epsilon^{3} V_{3}+\cdots\right)^{\epsilon}, \\
& \epsilon \equiv(1 /(n+1), 0 \leq \eta \leq 1,
\end{aligned}
$$

$$
x=\epsilon \cdot \eta \cdot\left(t^{1=m+m / \epsilon}\right)^{1 / 2} \cdot\left((1 / m) \cdot\left(\phi_{0}+\epsilon \phi_{1}+\epsilon^{2} \phi_{2}\right)+\cdots\right)^{1 / 2} .
$$

The location of the front is given by $\eta=1$ as

$$
x(\text { front })=F(t)=\epsilon \cdot\left(t^{m n+1}\right)^{1 / 2} \cdot\left((1 / m)\left(\phi_{0}+\epsilon \phi_{1}+\epsilon^{2} \phi_{2}\right)^{1 / 2} .\right.
$$

The $\phi$ 's and $V$ 's are defined in (18) and (17) respectively.

Comparison. The analytic form of the solution (19)-(21) obtained through the perturbation scheme described above was compared with some numerically computed solutions. The numerical scheme used for these comparisons is based on finite elements, employing Hermitian polynomials as the basis functions. As shown by van Genuchten [13], this scheme generated very accurate profiles for a number of infiltration experiments. Owing to the nonlinearity in the governing equation, especially for the larger $m$ and $n$ values, different spatial discretizations were used in the numerical solution leading to a reasonably accurate determination of the sharp concentration fronts.

Figs. 1 and 2 describe these results. In Figs. $1 a, b, c$, the curves are drawn for the times $t$ $=0.6,0.8$ and 1.0. In Figs. $2 a, 2 b$, the successive perturbation approximations are shown at $t=0.8$ and $t=1.0$. Several values of $m$ and $n$ were selected, and the figures also indicate the values of the pairs $(m, n)$ corresponding to each graph. It is clear that the agreement between the two results is quite good.

Appendix. A specialized ( $m=0)$ form of (3) has proved very effective in the construction of solutions to certain problems of moisture flow in soils (Babu [18]). The relation (3) may be generalized to

$$
\int_{0}^{F(t)} \theta(x, t) \cdot x \cdot d x=\int_{0}^{t} d \xi \int_{0}^{f(\xi)} D(\theta) d \theta
$$



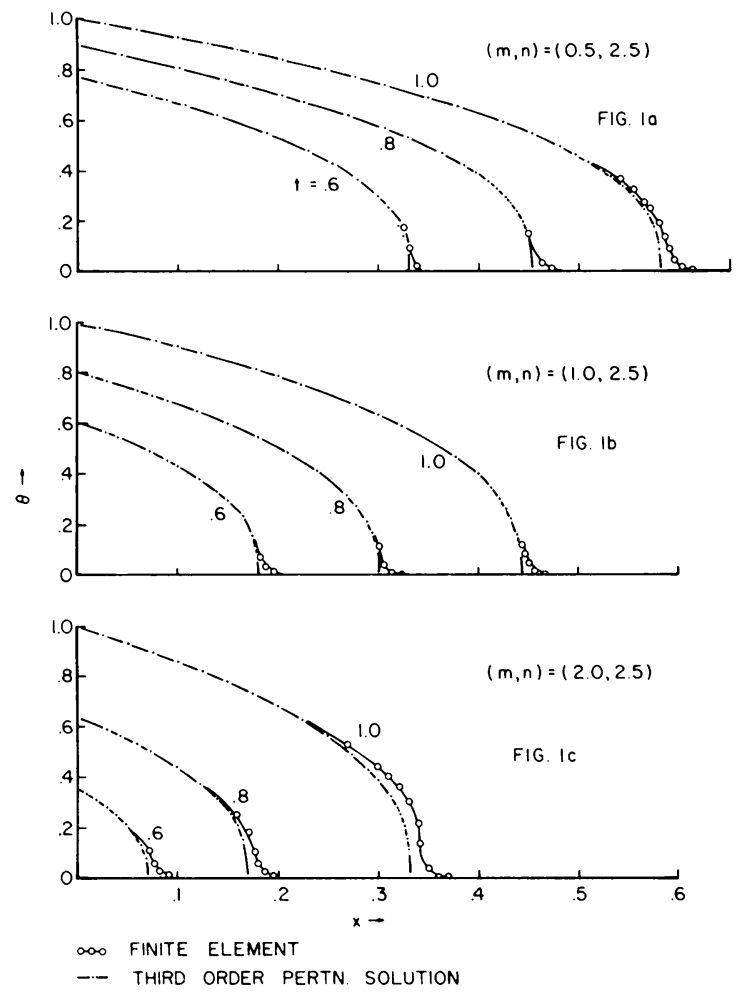

Fici. 1. Comparison of solutions for $\theta(x, t)$ at dimensionless times $0.6,0.8$ and 1.0. Perturbation solution (- - - ) computed to third order is compared with a numerical finite-element solution $(-0-0-)$. Results of changing diffusivity $\left(\theta^{n}\right)$ and boundary concentration $\left(t^{m}\right)$ are indicated by the three figures $(a, b, c)$. Values $(m, n)=(0.5$, $2.5),(1.0,2.5),(2.0,2.5)$.

where $f(t)=\theta(0, t)$ represents the concentration at the supply point $x=0$. If it is next assumed, after appropriate normalizations, that $f(t) \leq 1$, the characteristically nonlinear $D(\theta)$ defines a small number

$$
\epsilon \equiv \int_{0}^{1} D(\theta) d \theta \geq \int_{0}^{f(t)} D(\theta) d \theta
$$

leading to

$$
\int_{0}^{F(t)} \theta(x, t) \cdot x \cdot d x=\int_{0}^{t} d \xi \int_{0}^{f(\xi)} D(\theta) d \theta \equiv I(t, \epsilon),
$$

say. Clearly $I(t, \epsilon) \leq \epsilon \cdot t$, giving rise to a rough estimate

$$
I \equiv I(t, \epsilon)=O(\epsilon t) .
$$

Depending on the specific structure of $f(t)$, it may happen that the quantity $I$ is of a much smaller order of magnitude than $O(\epsilon t)$. Indeed, for the present problem, an exponentially large negative order was obtained:

$$
\left.I=O\left[\epsilon^{2} \cdot \exp (m \cdot \ln t) / \epsilon\right)\right]
$$



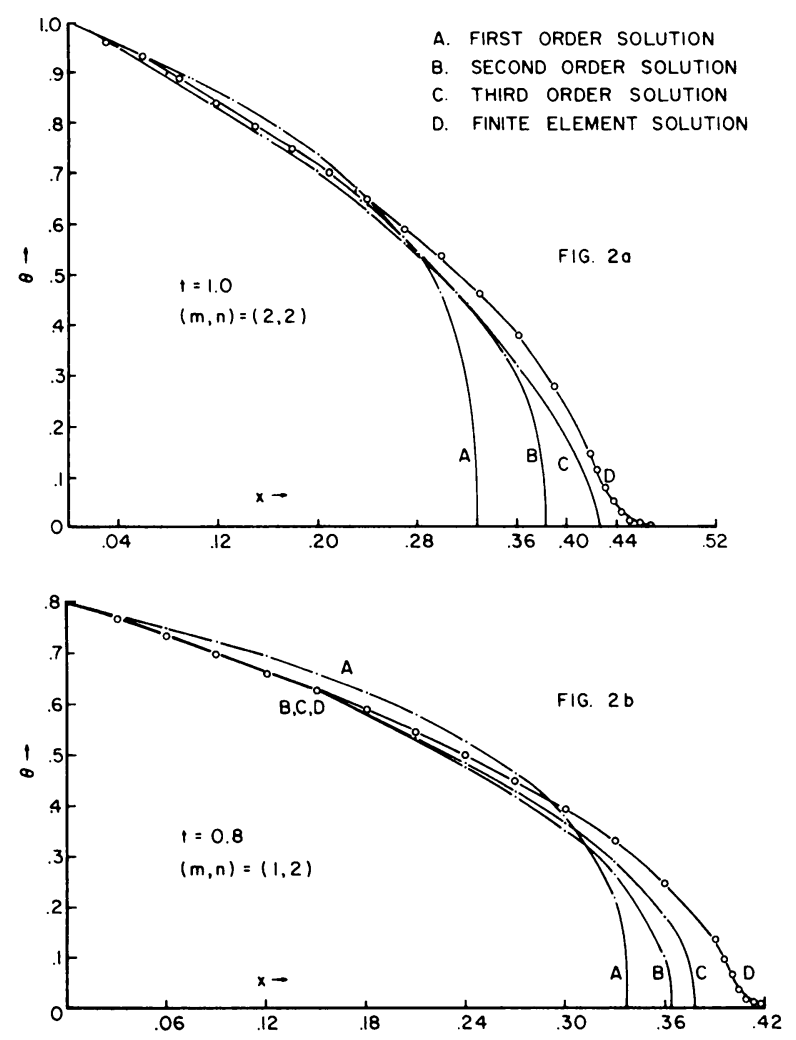

FIG. 2. Successive approximations up to third-order terms in the perturbation solution are graphed along with finite-element solution for a fixed time. Figures $(a, b)$ correspond to $(t ; m, n)=(1.0 ; 2,2)$ and $(0.8 ; 1,2)$.

Thus, from (24) follow two useful estimates. If $f(t)$ is monotonic increasing

$$
x=O\left((I / f(t))^{1 / 2}\right), \quad F(t)=O\left((I / f(t))^{1 / 2}\right) .
$$

If $f(t)$ is oscillatory, considering a mean value of $\theta(x, t)$,

$$
x=0\left((I(t, \epsilon))^{1 / 2}\right), \quad F(t)=O\left((I(t, \epsilon))^{1 / 2}\right) .
$$

The relations (26) and $\left(26^{1}\right)$ serve to estimate the location of the front, and the extent of the (material) transport at any given time. Additionally, a proper scaling of the variable $x$ introduces a small number $\epsilon$ into the mathematical formulation. The choice of $\epsilon$ as a parameter of perturbation is thus a natural and preferred one in this type of problem. The higher the degree of nonlinearity in $D(\theta)$, the sharper the estimates will come out. by

Note. Pattle's [3] solution for an instantaneous point source in the medium is given

$$
\begin{aligned}
D & =D_{0}\left(C / C_{0}\right)^{n}, \quad n>0, \\
C & =C_{0} \cdot\left(t / t_{0}\right)^{-s} /(s n+2) \cdot\left[1-\left(r^{2} / r_{1}{ }^{2}\right)\right]^{1 / n}, \quad t>0, \quad r^{2} \leq r_{1}^{2}, \\
& =0, \quad r^{2} \geq r_{1}^{2},
\end{aligned}
$$


with $r_{1}=r_{0}\left(t / t_{0}\right)^{1 / n(s n+2)} ; s=$ number of space dimensions. Upon setting $m=-\epsilon /(1+\epsilon)$, Eq. (5) simplifies to

$$
\left(d^{2} V / d \eta^{2}\right)=\epsilon \cdot \phi \cdot(d / d \eta)\left(\eta \cdot V^{\epsilon}\right)
$$

Upon integrating this equation with $V=(d V / d \eta)=0$ at $\eta=1$, an exact closed-form solution emerges:

$$
\begin{aligned}
V(\eta) & \propto\left(1-\eta^{2}\right)^{1 /(1-\epsilon)}, \\
\theta & \propto t^{m} \cdot\left(1-\eta^{2}\right)^{\epsilon /(1-\epsilon)} .
\end{aligned}
$$

It is obvious that, for $s=1$, the respective expressions for $C$ and $\theta$ in (27) and (28) are identical. For this particular value of $m$, it was possible to integrate Eq. (5) completely.

\section{REFERENCES}

[1] L. F. Shampine, Concentration-dependent diffusion. 2. Singular problems, Quart. Appl. Math 31, 287-293 (1973)

[2] K. Reichardt, D. R. Nielsen and J. W. Biggar, Scaling of horizontal infiltration into homogeneous soils, Soil Sc. Soc. Am. Proc. 36, 241-245 (1972)

[3] R. E. Pattle, "Diffusion from an instantaneous point source with concentration-dependent coefficient", Quart. J. Mech. Appl. Math. 12, 407-409 (1959)

[4] D. G. Aronson, Regularity properties of flows through porous media, SIAM 17, 461-467 (1969)

[5] L. A. Peleiter, Asymptotic behaviour of solutions of the porous medium equations, SIAM 21, 542-551 (1973)

[6] C. Atkinson and C. W. Jones, Similarity solution in some nonlinear diffusion problems and boundary layer flow of pseudo plastic fluid, Quart. J. Mech. Appl. Math. 27, 193-211 (1974)

[7] G. H. Pimbeley, Jr., Wave solutions travelling along quadratic paths for equation $U_{t}-\left(k(u) u_{x}\right)_{x}=0$, Quart. Appl. Math. 35, 129-138 (1977)

[8] J. R. Philip, Theory of infiltration, Adv. in Hydroscience 5, 215-296 (1969)

[9] J. R. Philip, Recent progress in the solution of the nonlinear diffusion equation, Soil Science 117, 257-264 (1974)

[10] G. Ashcroft, D. R. Marsh, D. D. Evans and L. Boersma, Numerical methods for solving the diffusion equation. 1. Horizontal flow in semi-infinite media, Soil Sci. Soc. Am. Proc. 26, 522-525 (1962)

[11] S. P. Neuman, Finite element computer programs for flow in saturated-unsaturated media, second annual report no. A 10-SWC-77, Hydr. Eng. Lab. Technion, Haifa, 1972

[12] H. N. Hayhoe, Study of the relative efficiency of finite difference and Galerkin techniques for modelling soil water transfer, Water Resources Res. 14, 97-102 (1978)

[13] M. Th. van Genuchten, Numerical solutions of the one-dimensional saturated-unsaturated flow equation, Water Resources Program, Research Report 78-WR-9, Dept. of Civil Engineering, Princeton University, 1978

[14a] W. Brutsaert, More on an approximate solution for the nonlinear diffusion equation. Water Resources Res. 10, 1251-1252 (1974)

[14b] W. Brutsaert and R. N. Weisman, Comparison of solutions of a nonlinear diffusion equation, Water Resources Res. 6, 642-644 (1970)

[15] M. A. Heaslet and A. Alksne, Diffusion from a fixed surface with a concentration-dependent coefficient, J. SIAM 9, 584-596 (1961)

[16] J.-Y. Parlange, Theory of water movement in soils. 1. One-dimensional absorption. Soil Science 111, 134-137 (1971)

[17] D. K. Babu, Infiltration analysis and perturbation methods. 1. Absorption with exponential diffusivity, Water Resources Res. 12, 89-93 (1976)

[18] D. K. Babu, Infiltration analysis and perturbation methods. 2. Horizontal absorption, Water Resources Res. 12, 1013-1018 (1976)

[19] D. K. Babu, Infiltration analysis and perturbation methods. 3. Vertical infiltration. Water Resources Res. 12, 1019-1024 (1976)

[20] J.-Y. Parlange and D. K. Babu, A comparison of techniques for solving the diffusion equation with an exponential diffusivity, Water Resources Res. 12, 1317-1318 (1976) 
[21] J.-Y. Parlange and D. K. Babu, On solving the nonlinear diffusion equation-a comparison of perturbation, iterative and optimal techniques for an arbitrary diffusion equation, Water Resources Res. 13, 213-214 (1977)

[22] Don Kirkham and W. L. Powers, Advanced soil physics, Wiley Interscience, 1972, pp. 87-95

[23] A. H. Nayfeh, Perturbation methods, Wiley Interscience, 1973

[24] R. E. O'Malley, Jr., Singular perturbation analysis for ordinary differential equations (lecture notes), Communications of the Mathematical Institute, Rijksuniversiteit Utrecht-5-1977 (The Netherlands)

[25] N. Krylov and N. Bogoliuboff, Introduction to nonlinear mechanics, Princeton University Press, 1947

[26] M. Van Dyke, Perturbation methods in fluid mechanics, The Parabolic Press, Stanford, California, 1975

[27] Asymptotic methods and singular perturbations, SIAM-AMS Proceedings X, American Math. Society, Providence, Rhode Island, 1976 